Articole

\title{
LE RÔLE DE LA RHÉTORIQUE DANS LE DÉVELOPPEMENT DE LA PENSÉE CRITIQUE DU DROIT
}

\section{THE ROLE OF RHETORIC IN THE DEVELOPMENT OF CRITICAL THINKING OF LAW}

\author{
DOI: $10.24193 /$ SUBBiur.63(2018).3.9 \\ Published Online: 2018-12-17 \\ Published Print: 2018-12-17
}

\section{Amar LAIDANI*}

\begin{abstract}
:
Rhetoric has accompanied the science of Law since its birth. This art has contributed to the birth of the Philosophy of Law and Critical Thinking in Law. Rhetoric is intrinsically linked to criticism. It is an approach which consists of submitting every argument to judgment according to the method of antilogy. Rhetoric is, therefore, a pedagogy of criticism.

Thanks to Perelman's work, the potentialities of rhetoric has been rediscovered by lawyers. That said, the modern Critical Legal Theories have underestimated the critical potential of rhetoric. We will try to explain in our article the origin of the fracture between the art of rhetoric and critical thinking and will illustrate how Perelman's New Rhetoric has recomposed this fracture.

Keywords: rhetoric, topical, dialectic, critical thought.

\section{Résumé :}

La rhétorique a accompagné le droit dès sa naissance. Cet art a contribué à la naissance de la philosophie du droit et de la pensée critique en droit. La méthode rhétorique est une méthode intrinsèquement critique. C'est une démarche qui consiste à soumettre tout argument à un jugement selon la méthode des antilogies. La rhétorique est ainsi une pédagogie du sens critique.
\end{abstract}


Grâce aux travaux de Perelman, les potentialités de la rhétorique ont fait l'objet d'une redécouverte de la part des juristes. Cela dit, la portée critique de la rhétorique a été sous-évaluée par les théories critiques du droit. Nous essayerons d'expliquer par notre article l'origine de la fracture entre l'art de la rhétorique et la pensée critique et nous illustrerons la façon dont la nouvelle rhétorique de Perelman a recomposé cette fracture.

Mots clés : rhétorique, topique, dialectique, pensée critique.

\section{Introduction}

La critique est un élément consubstantiel au droit. En Occident le droit est né durant l'Antiquité grecque sous la forme du procès et, plus généralement, de débat. C'est dans ce même contexte qu'est née la rhétorique qui est un art de la persuasion par le biais d'arguments. Un art - qui tout comme le droit - est né dans les tribunaux.

A partir des années 1960 nous assistons à un retour en force de la rhétorique et de la topique au sein de la philosophie du droit. Cette redécouverte d'une logique juridique prémoderne s'explique en partie par la crise qu'a connue le positivisme juridique kelsenien durant l'après-guerre ainsi que par la réapparition de la raison pratique au sein de la philosophie du droit.

La redécouverte de la topique et de la rhétorique par Perelman a eu pour pendant la mise en valeur de l'utilité du débat en droit. Les tribunaux, en tant que lieux où s'exerce une critique du droit par le biais d'arguments rationnels, sont devenus à nouveau une source d'inspiration pour la théorie du droit.

Le potentiel critique de la rhétorique a été sous-évalué par les écoles critiques du droit et cela bien que la rhétorique soit à l'origine même de la pensée critique en droit. La fracture entre la pensée critique et la rhétorique 
a des origines lointaines. Dans notre article, nous allons illustrer les raisons historiques d'une telle rupture ainsi que démontrer comment et pour quelle raison la rhétorique peut se révéler un outil formidable de critique du droit. Nous débuterons au chapitre 2 par une analyse historique sur le rôle qu'a joué l'art de la rhétorique dans l'histoire de la pensée juridique dès l'antiquité grecque et romaine. Nous analyserons par la suite au chapitre 3 les raisons qui ont porté à la rupture qui s'est consommée entre la rhétorique et la pensée critique à partir du XVIe siècle. Nous nous pencherons sur l'école moderne du droit naturel, ainsi que sur la façon dont Vico, par le bais de la rhétorique, a mené une contre-critique à la pensée critique. Enfin nous verrons au chapitre 4 de quelle façon la nouvelle rhétorique de Perelman a représenté une véritable rhétorique critique qui a réconcilié la rhétorique avec la dialectique et la pensée critique. Nous verrons ainsi comment cette rhétorique critique représente un dépassement de la pensée problématique de Viehweg et comment cette nouvelle rhétorique présente plusieurs analogies avec la critique du droit de Habermas.

\section{La rhétorique à l'origine de la critique en droit}

\subsubsection{A. La rhétorique à l'origine de la pensée critique dans la Grèce antique}

Le mot critique vient du verbe grec krino/krinein qui signifie « discerner » ainsi que « juger ». La notion de critique est ainsi strictement liée au monde du droit. C'est un élément qui accompagne le droit dès sa naissance. Dès son apparition au sein de la tradition occidentale, durant l'antiquité grecque et romaine, le droit s'est manifesté sous la forme du procès ${ }^{1}$. Villey a défini le procès de l'antiquité comme étant une « controverse 
d'opinions », en ajoutant que la controverse a été le « [...] berceau du droit $»^{2}$.

La littérature classique et la philosophie grecque ont maintes fois fait référence au monde du droit. Les scènes de procès-arbitrage étaient omniprésentes dans les œuvres poétiques et théâtrales de l'antiquité. Le jugement y est décrit comme moyen d'apaisement des conflits. Nous avons ainsi l'exemple de l'épisode du bouclier d'Achille décrit dans l'Iliade (XVIII, 50). Cette idée est omniprésente au sein des tragédies grecques telles que l'Antigone de Sophocle ou les Euménides d'Eschyle. Les dialogues de ces tragédies étaient structurés de façon très semblable aux débats qui se déroulaient durant les procès publics 3 .

Les procès publics qui se déroulaient dans les principales villes grecques avaient inspiré les philosophes présocratiques. Anaximandre utilisa la métaphore du procès pour décrire l'apeiron, ou bien l'origine de toutes les choses. Dans son unique fragment qui nous soit parvenu, Anaximandre affirme ainsi que :

«Toutes choses ont racine l'une dans l'autre et périssent l'une dans l'autre, selon la nécessité. Elles se rendent justice l'une à l'autre et se récompensent pour l'injustice conformément à l'ordre du temps "4.

Le philologue Werner Jaeger percevait dans ce fragment d'Anaximandre une influence du monde du droit. Plus précisément celle des procès qui se déroulaient au sein des cités-État ioniennes auxquelles Anaximandre avait assisté. Jaeger souligne à ce propos :

«Il faut se figurer ce qu'est une cité-État ionienne. Elle comporte la place du marché où les requêtes sont entendues et les jugements rendus : le juge est assis sur un banc pour décider des amendes qu'il convient de payer. Le juge est le temps $\gg 5$.

Cela dit la justice à laquelle fait référence Anaximandre est une justice cosmique. Ce n'est pas la justice du monde d’ici-bas ${ }^{6}$. En ce sens, l’idée du 
conflit -pomélos comme berceau de la justice était encore plus marquée dans la philosophie d'Héraclite, ce dernier affirmait dans son fameux fragment :

« Il faut savoir que le combat est universel, que la justice est une lutte et que toutes les choses naissent selon la lutte et la nécessité (80) »7.

Le conflit est ainsi le moteur de toute chose, à savoir que la vérité ne peut surgir qu'à partir d'un conflit d'opinions.

Comme nous le voyons, les jugements qui se tenaient devant des assemblées publiques avaient profondément marqué la culture de la Grèce antique. Les procès publics ont été d'une certaine manière les berceaux de la logique discursive et argumentative. Pour remporter le débat, il fallait repérer des bons arguments et savoir les exposer de façon convaincante. Ces capacités ne pouvaient pas être laissées à la simple improvisation, elles nécessitaient une technique, voire une méthodologie. Ce sera dans ce contexte que va naitre l'art de la rhétorique.

Selon les sources classiques, les deux premiers traités de rhétorique ont été rédigés en Sicile. Les auteurs de ces traités étaient le philosophe Empédocle d'Agrigente et un personnage du nom de Corax ${ }^{8}$. Ce dernier avait gagné la réputation de grand orateur durant un procès qui se déroula à Syracuse durant lequel il siégeait sur le banc des accusés. Dans ce fameux procès, Corax avait réussi à se faire acquitter de l'accusation de complaisance avec le régime de l'ancien tyran qui venait d'être déchu. Par la suite, au vu de sa réputation de grand orateur acquise grâce au procès de Syracuse, Corax enseigna son art à plusieurs disciples, dont le plus célèbre fut Tisias. Ce dernier enseigna à son tour l'art de la rhétorique au sophiste Gorgias qui, à son tour, diffusa cet art auprès des sophistes. Platon dans le Phèdre (267 a, 273) et Aristote dans La Rhétorique (II, 1402 a 18) affirment que cette rhétorique presophistique ne visait pas à la connaissance d'une vérité 
absolue (aletheia) mais plutôt à celle du vraisemblable (eikos). Ce qui en découlaient que tout propos pouvait à tout moment être falsifié par un propos contraire.

La rhétorique devenant une véritable marque de fabrique des sophistes tels que Protagoras et Gorgias, ces derniers ayant une vision relativiste du concept de vérité (relativisme ontologique) ainsi que de l'éthique (relativisme étique), voyaient en la rhétorique la technique idéale pour développer leur philosophie centrée sur le discours. La manière de procéder des sophistes était celle des doubles discours (dissoi logoi). Cette technique permettait aux sophistes de plaider pour une thèse ainsi que pour son contraire. Cette façon de procéder les avaient aidés à développer un fort sens critique. Cela pour la raison qu'une telle démarche poussait les sophistes à prendre en considération le point de vue d'autrui avant de le critiquer.

Les sophistes étaient avant tout des enseignants qui n’inculquaient pas des notions à leurs étudiants mais qui leur enseignaient plutôt une méthode leur permettant d'exposer leurs idées de façon efficace. Les cours des sophistes consistaient en des simulations de plaidoyers fictifs durant lesquels les étudiants devaient être capables de plaider pour une cause et son contraire$^{9}$. Le philosophe Sextus Empiricus qualifia la pédagogie du sophiste Gorgias comme étant avant tout l'enseignement du sens critique, à savoir Kritérion $^{10}$ ou, mieux, le moyen de juger ${ }^{11}$.

Les sophistes vont développer la première critique du droit et cela le biais de la rhétorique. Les sophistes tels que Gorgias et Protagoras ne croyaient pas en l'existence d'un lien intrinsèque entre l'idée de réalité (Physis), le langage (Logos) et les lois (Nomos) ${ }^{12}$. Cette unité avait été soutenue jusque-là par les philosophes présocratiques ainsi que les pythagoriciens. Le droit, tout comme le langage, était pour les sophistes le 
produit de conventions humaines. La loi positive est désormais perçue comme étant distincte aussi bien de la loi naturelle que de la loi divine ${ }^{13}$. Sur de tels fondements, les sophistes ont établi leur conception clairement antidogmatique du droit.

Protagoras affirmait que les lois humaines devaient être respectées seulement à partir du moment où celles-ci reflétaient un consensus social. C'est grâce à cette vision séculaire du droit que Protagoras développera les premières réflexions sur la fonction rééducative de la peine qu'a connues l'antiquité. La peine, contrairement à la vengeance, n'avait pas pour finalité d'apaiser la colère des morts mais de corriger le coupable et d'inhiber un certain type de comportement. Cette idée sera reprise par la suite par Platon dans le Protagoras (324 a, b) et par Sénèque dans le De Ira (I, 19).

La négation de tout attribut rationnel aux principes ontologiques du droit tels que l’idée de justice et de loi naturelle avait induit certains sophistes à identifier le droit avec les lois écrites. Celles-ci n'étaient à leurs yeux rien d'autre que le produit des rapports de force. Les principes tels que la justice n'étaient à leurs yeux que des trompe-l'œil qui occultaient les hiérarchies sociales. La critique du droit visait avant tout à dévoiler les rapports de force occultés par ces principes. La vision critique du droit des sophistes émerge dans la République de Platon. Plus précisément dans le débat qui opposa Socrate aux sophistes Thrasymaque et Critias autour de la notion de justice. Ces derniers soutenaient que les lois n'étaient rien d'autre que des « masques » que les puissants avaient mis en place afin de cacher les rapports de force présents dans la société (338cl-339a4). D’autres sophistes tels que Calliclés, dans d'autres dialogues platoniciens tels que le Gorgias (481b-56o b), affirmaient la thèse contraire, à savoir que le droit était une invention des 
faibles mises en place en guise de défense à l'égard des attaques des plus forts.

Ce relativisme gnoséologique et moral prôné par les sophistes avait été critiqué par Platon et Aristote. Dans les dialogues platoniciens, la critique des sophistes s'accompagnait d'une critique de l'art de la rhétorique. Par exemple, dans les dialogues du Gorgias et de l'Hippias, ainsi que du Protagoras, les sophistes sont présentés comme de dangereux charlatans. Dans le dialogue de Phèdre, Platon avait adopté un jugement plus nuancé à l'égard de la rhétorique. Ce dernier distingue ainsi entre une bonne et une mauvaise rhétorique. Cela dit, la bonne rhétorique selon Platon n'est pas praticable dans le monde d'ici-bas et peut exister uniquement dans le monde des idées ${ }^{14}$. L'unique méthode discursive praticable dans le monde réel est, toujours selon Platon, la dialectique telle qu'elle avait été pratiquée par Socrate. Dans le dialogue du Phèdre, Platon prend soin de séparer de façon tranchée la dialectique de la rhétorique. La dialectique devient ainsi la méthode logique par excellence. Cette consécration de la dialectique aux dépens du déclassement de la rhétorique marqua à tout jamais l'histoire de la pensée occidentale. Cela aura pour conséquence une première rupture entre la pensée critique et la rhétorique. Comme nous le verrons, cette idée sera reprise par la logique stoïcienne et par la dialectique médiévale.

Dans le dialogue de Phèdre, Socrate dicte la méthodologie que doit utiliser tout dialecticien. Une méthode qui consiste en deux opérations, la division et le rassemblement. La première, connue sous le nom de diairesis, consiste à définir l'objet de la discussion en isolant tous les éléments qui caractérisent l'objet en question (ousia). Cette opération avait pour but de réduire les ambiguïtés du langage, de façon à éviter que celles-ci soient exploitées par les rhéteurs. (266 b 3-c 1). La deuxième opération est la 
synapsis ou le rassemblement qui consiste à chercher l'unité d'une multiplicité (266 b 3-c 1).

La dialectique de Platon est une méthodologie critique, voire une théorie du jugement qui, afin d'éviter les doubles discours des sophistes, se concentre non pas sur la quête d'arguments (topique), mais sur la recherche de définitions précises. C'est une démarche qui, bien que reposant clairement sur le dialogue, évite les antilogies des sophistes. Ces dernières sont considérées par Socrate comme étant une simple démarche éristique, à savoir un procédé fondé sur la dispute (éris) qui, pour cela, ne peut pas être qualifié de méthodologie.

Contrairement à Platon, Aristote ne fera pas le choix d'opposer la dialectique à la rhétorique et encore moins de subordonner la rhétorique à la dialectique. Selon Aristote la rhétorique est plutôt le pendant (antistrophos) de la dialectique. Aristote affirme clairement cette idée dans l'exorde de sa Rhétorique ${ }^{15}$ :

« La rhétorique se rattache à la dialectique. L'une comme l'autre s'occupe de certaines choses qui, communes par quelque point à tout le monde, peuvent être connues sans le secours d'aucune science déterminée (I, 1) ».

Contrairement à Socrate, Aristote soutient que la dialectique, tout comme la rhétorique, repose sur le concept de dispute (eris) et de pólemos. Cela émerge de façon plutôt claire dans son ouvrage intitulé La rhétorique où Aristote affirme :

« Aussi tout le monde, plus ou moins les pratique l'une et l'autre; tout le monde, dans une certaine mesure, essaie de combattre et de soutenir une raison, de défendre, d'accuser $(I, 1)$ ».

La logique aristotélicienne est un système logique ouvert qui inclut des formes de raisonnement qui ne reposent pas sur le principe de noncontradiction, ni sur la logique inductive-déductive et ni sur des procédés 
apodictiques. En un mot la logique d'Aristote ne conçoit pas uniquement les procédés des sciences pures telles que les mathématiques.

Le système aristotélicien prévoit deux types de raisonnement. Le premier est propre aux sciences exactes, à savoir le syllogisme scientifique. Ce type de raisonnement est décrit dans ses premiers et seconds Livres Analytique. Le syllogisme scientifique est un raisonnement dont les prémisses (ou axiomes) demeurent certaines et par conséquent elles ne doivent pas être prouvées. A côté de ce type de syllogisme, Aristote prévoit la présence d'un syllogisme dialectique. Ce raisonnement est décrit dans son traité intitulé Les Topiques. Ce type de raisonnement repose sur des prémisses qui n’ont pas le niveau de certitude des vérités scientifiques. Ce sont des raisonnements qui se fondent sur des vérités partagées qu'Aristote définit de lieux communs ou tópoi (VIII, 14, 164 b). Dans ses Topiques Aristote analyse et expose une série d'arguments, ou mieux, de lieux communs qui pouvaient être utiles aux dialecticiens.

Contrairement à la dialectique socratique et platonicienne, la dialectique aristotélicienne est une théorie du jugement ayant pour opération première non pas la quête de définitions mais plutôt la recherche d'arguments. La dialectique aristotélicienne devait se dérouler en trois opérations. La première consistait en une quête d'arguments (inventioeuresis) qui puissent résister aux attaques des adversaires (praemunitio). Par la suite, les arguments devaient être disposés (dispositio) et exposés (elocutio) de la façon la plus persuasive possible (VIII, 155 b). Pour conclure, la dialectique d'Arioste repose dans toutes ses phases sur un raisonnement de type discursif et argumentatif. Cela la rapproche ultérieurement à la rhétorique. Ce rapprochement est d'autant plus évident qu'Arioste qualifie, dans son traité intitulé $L a$ Rhétorique, le raisonnement rhétorique 
(L'enthymème) comme étant une « sorte de syllogisme » pour la raison qu'il [...] appartient tout aussi bien à la dialectique, prise dans son ensemble ou dans quelqu'une de ses parties, d'examiner tout ce qui se rattache au syllogisme $(\mathrm{I}, 11) »$.

La rhétorique et la dialectique étaient selon Arioste des technè qui n'avaient pas pour but de démontrer des vérités absolues. Celles-ci visaient plutôt à des vérités partagées. En un mot nous somme dans le registre du vraisemblable. Cela dit Aristote prend ses distances à l'égard du relativisme gnoséologique et éthique des sophistes. Ainsi Aristote dans ses Réfutations sophistiques critique les paralogismes fallacieux utilisés par les sophistes en décrivant toute une série de raisonnements euristiques utilisés par les sophistes afin de tromper leurs adversaires. Ce type de raisonnement condamnait à l'erreur quiconque les utilisait. La question de l'éthique de la discussion est affrontée dans La Rhétorique. Aristote rattache aux trois types principaux de rhétorique trois valeurs différentes. Le délibérative a comme valeur l'utile, le judicaire la justice, l'épidictique le beau et le bon. Aristote affirme que :

« La rhétorique est utile, d'abord, parce que le vrai et le juste sont naturellement préférables à leurs contraires $[\ldots](\mathrm{I}, 12) »$.

Aristote développe dans son Livre de l'interprétation une ultérieure théorie du jugement (outre la dialectique) et donc de la critique qui réconcilie la pensée critique avec les antilogies utilisées par les rhéteurs. Cette réconciliation est présente dans un type particulier de jugement qu'Aristote qualifie de «propositions subcontraires ». Ce type de jugement prévoit que deux propositions peuvent porter deux jugements opposés sur une même question tout en demeurant également vraies. Contrairement au jugement fondé sur le principe de contradiction, la proposition subcontraire prévoit 
que la vérité d'une proposition n’implique pas à priori la fausseté de la proposition contraire. Ainsi Aristote reconnait une dignité philosophique à ce que Popper qualifiera dans son épistémologie de principe de falsifiabilité. En conclusion ce type de jugement prévoit que deux prépositions peuvent également être à priori vraies avant que n'intervienne à posteriori la falsification de l'une par l'autre. Fallone a très pertinemment vu en ce type de jugement une légitimation sur le plan logique du mécanisme du procès accusatoire, c'est-à-dire le droit à la preuve contraire ${ }^{16}$. Ainsi Aristote développe une théorie de la critique qui s'harmonise avec les procédés propres au monde du droit. La vérité qui l'emporte sera la vérité qui sera la mieux prouvée, à savoir la mieux argumentée. Par conséquent Aristote accorde une grande importance à la notion de preuve, cela émerge de façon claire dans La Rhétorique :

« La méthode, en matière de rhétorique repose évidemment sur les preuves. La preuve est une démonstration (car si nous admettons une preuve, c'est surtout lorsque nous supposons qu'il y a eu une démonstration). D'autre part, la démonstration oratoire c'est l'enthymème, qui est, en résumé la preuve par excellence (I, 11) »,

Par la suite Aristote affirme qu'au sein des procès la preuve par témoins demeure le moyen probatoire par excellence (I, 13-19). Cela vaut du moment où le témoignage est spontané. Aristote se montre ainsi critique à l'égard de la véridicité du témoignage arraché par le biais de la torture.

" Les gens contraints par la torture, dira-t-on, ne disent pas moins des mensonges que des choses vraies [...] (I, 26, 1377 a)».

La preuve par témoin tout comme la preuve rhétorique repose sur le principe de falsification. Pour les Grecs, tout comme pour les Romains, la preuve n'avait pas uniquement la fonction de prouver l'existence d'un fait. La preuve servait, avant tout, à invalider les propositions contraires. Cette idée sera reprise par le rhéteur romain Cicéron qui, dans le De partitionibus 
oratoriae (23 et suivantes), soutient l'idée selon laquelle l'utilité d'une preuve (dans le sens d'argument) doit être recherchée dans sa capacité d’invalider les thèses des adversaires. Cette technique était connue par les romains sous le nom de premunitio. Ce terme provient du grec prokatalapsis. Dans la terminologie militaire, cela signifiait anticiper de façon préventive les critiques des adversaires. Selon Aristote les dialecticiens ainsi que les rhéteurs se devaient de tester et par conséquent de soumettre à une critique les arguments qu'ils ont l'intention de déployer. Par conséquent il faut falsifier ses propres arguments. Aristote conseille cette technique dans le douzième chapitre de La Rhétorique, cela dans les termes suivants :

« Il faut, de plus être en état de plaider le contraire de sa proposition, comme il arrive en fait de syllogisme, non pas dans le but de pratiquer l'un et l'autre (le non vrai et le non juste), car il ne faut pas conseiller le mal, mais pour ne pas ignorer ce qu'il en est, et afin que, si quelque autre orateur voulait discourir au détriment de la justice, nous soyons nous-même en mesure de détruire ses arguments $(\mathrm{I}, 12) »$.

Cette technique sera utilisée par Cicéron (De l'orateur, III, 204 s) et Quintilien (Orateur IX, 2, 16). Avec Aristote la dialectique et la pensée critique se réconcilient avec la rhétorique. Cette réconciliation fut encore plus marquée au sein du droit romain.

\subsubsection{B. La rhétorique romaine à l'origine de la philosophie du droit}

Pour les rhéteurs romains la rhétorique était une véritable méthodologie. La rhétorique servait à l'étude et à l'interprétation ainsi qu'à la critique du droit «Ius ». Un droit qui avait obtenu à Rome le statut de science $^{17}$. Cela grâce à des ouvrages de jurisprudence rédigés par des juristes laïcs (non appartenant à la caste sacerdotale) tels que l'Enchiridion du jurisconsulte Pomponius ainsi que l'œuvre perdue de Scevola et de Servius Suplicius $^{18}$. 
La naissance de cette nouvelle science a fait surgir le problème d'une méthode. Dans un premier temps le jurisconsulte Scevola s'était tourné vers la méthode de la diairesis ${ }^{19}$. Une méthode qui, comme nous l'avons vu plus haut, avait été élaborée dans le dialogue de Phèdre. Par la suite, le jurisconsulte Servius Sulpicius Rufus se tournera vers la rhétorique, une rhétorique qui se complétait avec la dialectique. L'idée d'une complémentarité entre la rhétorique et la dialectique était présente dans l'ouvrage d'Hermagoras ainsi que dans le traité anonyme de La Rhétorique ad Hérennius ${ }^{20}$. Servius Sulpicius Rufus était une des figures qui influença le plus la pensée juridique de Cicéron. Ce dernier rendra hommage à Servius Sulpicius Rufus dans sa neuvième Philippique $(I X, 5)$. Les rhéteurs romains tels que Cicéron, Quintilien et Sénèque, cherchèrent sous l’influence de la philosophie stoïcienne à réconcilier l'art de la rhétorique avec la philosophie platonicienne et aristotélicienne. Cicéron réalisa cette conciliation à travers la théorisation d'une rhétorique au service de la vérité. Une vérité qui était avant tout le résultat d'un consensus entre les théories les plus importantes de son temps. La rhétorique romaine (surtout cicéronienne) fournira à la science du droit (la jurisprudence ou iuris-prudentia) une méthode d'analyse, de critique et d'enseignement critique. En un mot la rhétorique a fourni une logique au droit. Michel explique ainsi :

« [...] pour Cicéron, toute discussion sur le droit implique la mise en œuvre d'une réflexion philosophique, qui même à propos des lois ou des édits particuliers, pose en général le problème du droit et de l'équité ${ }^{21}$.

Cette position amène Fassò à considérer Cicéron comme étant le premier philosophe du droit ${ }^{22}$. Une philosophie du droit qui nait de la réflexion des juristes sur leur propre science, connue désormais sous le nom de ius. Par conséquent, une philosophie du droit qui n'est pas, comme chez les Grecs, une réflexion du philosophe sur le droit, mais plutôt une réflexion 
que le juriste mène sur sa science en instrumentalisant pour cela les procédés que lui offre la philosophie. En un mot c'est une philosophie du droit créée par des juristes. Le droit peut se juger et critiquer par lui-même. Selon Cicéron, les juristes praticiens, par le fait qu'ils maitrisaient la rhétorique, était les interprètes privilégiés du droit. Michel ne manque pas de remarquer sur ce point que, selon Cicéron :

« Seul l'orateur est capable d'étudier correctement le droit, d'aborder ses difficultés dans l'esprit voulu. Tous les textes de Cicéron sont d'accord sur ce point ${ }^{23}$.

Dans son dialogue intitulé Des $\operatorname{lois}^{24}$, que nous pouvons considérer comme le premier traité de philosophie du droit écrit par un juriste, Cicéron s'interroge autour de l'origine du droit et expose ainsi sa propre vision du droit naturel. Le traité est structuré comme étant un dialogue-débat fictif qui se déroule entre deux personnages Atticus et Marcus. Ce dernier n'est rien d'autre que Cicéron. Les concepts traités sont le résultat d'un dialogue critique. C'est par le bais de la discussion et de la critique que Cicéron développe les concepts clés de sa philosophie du droit.

Dans ce dialogue Cicéron reprend l'idée stoïcienne d'une justice fondée sur la loi naturelle. Ainsi celle-ci trouve son origine dans la nature ainsi que dans la raison humaine. Cicéron définit la loi naturelle comme étant :

« [...] la raison suprême gravée en notre nature, qui prescrit ce que l'on doit faire et interdit ce qu'il faut éviter de faire $(\mathrm{I}, 6)$ ».

Contrairement à ce que soutenaient les sophistes, selon Cicéron le droit ne se résume pas aux lois écrites et aux coutumes. Pour la raison que : «[...] tenir pour juste tout ce qui est réglé par les institutions ou lois des peuples » demeure une « $[\ldots]$ absurdité, et la plus forte » $(\mathrm{I}, 15)$.

Cicéron précise que : 
«[...] si les volontés des peuples, les décrets des chefs de l'État, les sentences des juges fondaient le droit, le vol serait de droit ; l'adultère, les faux testaments seraient de droit, dès qu'on aurait l'appui des suffrages ou des votes de la multitude » $(\mathrm{I}, 16)$ ».

Toujours dans Les Lois, Cicéron applique au droit les vertus qu'Aristote avait accordées à la rhétorique judiciaire : « le juste et l'honnête sont désirables par eux-mêmes » (I, 18). Ceci étant, Cicéron n'a pas une vision irénique du droit. Ce dernier, bien qu'accordant une nature rationnelle aux principes tels que la justice et la loi naturelle, est conscient que le droit peut devenir une arme dans les mains d'un pouvoir tyrannique. Cette idée est résumée dans son ouvrage intitulé Des devoirs par la fameuse formule selon laquelle le meilleur des systèmes juridiques peut produire la pire injustice, à savoir - summum ius summa iniuria (I, 33).

Dans son ouvrage intitulé Les Topiques, Cicéron affronte la problématique de la méthodologie applicable à la science du droit. Selon Cicéron la rhétorique est placée à égalité vis-à-vis de la dialectique, si ce n'est même en supériorité. Michel précise sur ce point que :

« [...] la rhétorique, en introduisant la dialectique dans le droit, a contribué à l'ouvrir aux idées générales et à rendre possible son enseignement rationnel ${ }^{25}$.

Selon Cicéron, la rhétorique a besoin de la philosophie tout comme la philosophie de la rhétorique. Dans son traité intitulé De l'Orateur ${ }^{26}$ il affirme que malgré le fait que dans les œuvres de Platon le personnage du rhéteur soit « [...] fort maltraité » (IV), les ouvrages de philosophie demeurent à ses yeux « d'un grand secours pour l'éloquence [...] » (IV) et il conclut en adressant aux rhéteurs les paroles suivantes : 
« Nous ne pouvons en effet sans le secours de la philosophie, distinguer le genre et l'espèce, définir, diviser, discerner le vair le faux (IV) ».

Cicéron soutient que tout comme la rhétorique ne pourrait pas se passer de la philosophie, la dialectique ne pourrait pas ignorer la rhétorique et la topique. Pour ces raisons, dans le deuxième livre de ses Topiques, Cicéron critique la méthode dialectique utilisée par les stoïciens. Cela par le fait que celle-ci se concentre uniquement sur les définitions en délaissant la phase de l'invention (inventio) utilisée par les rhéteurs. Tout comme pour Aristote, Cicéron soutient que toute démarche dialectique ou critique doit préalablement être accompagnée par une topique. A savoir un repérage d'arguments- tópoi. Cette phase doit précéder la diairesis ou bien la quête des définitions. Ainsi Cicéron expose sa vision de la dialectique dans le deuxième chapitre de ses Topiques ${ }^{27}$ :

« Toute discussion régulière se divise en deux parties, l'invention et le raisonnement : pour l'une comme pour l'autre, Aristote est, selon moi, un excellent maître. Les stoïciens ne se sont occupés que de la dernière : ils ont enseigné avec soin tous les procédés du raisonnement, au moyen de cette science qu'ils nomment la Dialectique ; mais ils ont entièrement négligé l'invention ou la Topique, laquelle a, dans l'usage, bien plus d'importance, et doit, dans l'ordre naturel, passer avant la science du raisonnement. Pour moi, qui estime ces deux parties de la plus haute utilité, je me propose de les traiter l'une et l'autre si j'en ai le temps. (II) ».

Cicéron avait transcrit une série des arguments (topica) destinés aux juristes (105 b et suivantes) ainsi que des instruments méthodologiques (organa) qui puissent aider les jurisconsultes à appréhender leur science ${ }^{28}$. A partir de l'art de la topique et de la rhétorique, Cicéron développe une véritable herméneutique par le biais de laquelle ce dernier classe les catégories juridiques (oroi) ${ }^{29}$. Johannes Stroux avait remarqué que pour Cicéron la rhétorique était aussi une méthodologie d'interprétation du droit. Cicéron a développé sa doctrine des statuts à travers un type d'approche qui 
était propre à la pratique judiciaire, la manipulation des lois, des preuves et des arguments ${ }^{30}$.

Cicéron en harmonisant la rhétorique avec la dialectique avait permis l'enseignement critique du droit. La pédagogie offerte dans les écoles de rhétorique romaines aux futurs avocats inculquait le sens critique aux étudiants. Les écoles de rhétorique dispensaient un enseignement à partir de cas pratiques, connu sous le nom de controversiae. Ces dernières étaient des simulations de procès durant lesquelles l'étudiant devait être capable de plaider en faveur de deux parties différentes. En ce sens, les écoles de rhétorique romaines adoptaient une pédagogie qui se rapprochait de celle des sophistes grecs. Les étudiants apprenaient l'art de la topique à partir des Topiques d'Aristote et de Cicéron (loci argumentorum). Ce type d'enseignement avait exercé une influence très prononcée sur la littérature apologétique chrétienne, en particulier les œuvres rédigées par les Pères de l'Église originaires des colonies romaines d'Afrique. La plupart des pères de l'Église de la période pré-nicéenne tels que Tertullien, Arnobe et Lactance avaient, avant leur conversion, exercé la profession d'avocat. Par conséquent, ils avaient étudié la rhétorique. Cela leur avait permis de développer un fort sens critique qu'ils utiliseront dans leur œuvre apologétique afin de défendre les chrétiens qui firent l'objet de persécutions. Leur stratégie consistait à mettre le système judiciaire romain devant ses propres contradictions. Les apologies étaient structurées de façon similaire à des plaidoiries. En ce sens l'exemple le plus intéressant demeure l'Apologeticum de Tertullien. Ce dernier, dans sa prémunition, adopte une vision désenchantée des lois humaines. Tout comme les sophistes, Tertullien affirme dans son Apologeticum $^{31}$ que les lois humaines (et non pas divines) ont leurs racines dans les sociétés humaines et donc : 
« Les lois étant humaines peuvent être erronées [...] (IV, 5)».

Les lois sont le produit de leur époque. Par conséquent selon Tertullien, les lois du passé peuvent paraitre comme absurdes aux contemporains. Tertullien affirme :

« [...] il existait autrefois des lois qui permettaient aux créanciers de couper en morceaux les débiteurs condamnés ; d'un commun accord, cette loi cruelle (crudelitas) fut plus tard abolie (IV, 9) ».

Lactance, dans le cinquième livre apologétique intitulé les Institutions divines ${ }^{32}$, dénonce les différences entre les classes sociales au sein de la société romaine. La justice ne peut pas exister en absence de justice sociale. Lactance utilise la métaphore de la justice qui s'est envolée pour la raison :

« [...] qu'il y avait chez eux des hommes de conditions différentes et de toutes classes, allant des indigents aux riches, des humbles aux puissants, des simples particuliers enfin jusqu'aux très sublimes puissances des souverains $(\mathrm{V}, 14,19) »$.

Saint-Augustin, qui avait avant sa conversion enseigné l'art de la rhétorique, prit soin de séparer l'éloquence de la rhétorique ${ }^{33}$. L'éloquence devient un outil d'interprétation des Écritures, tandis que la rhétorique profane devient un instrument que le père de l'Église utilisait pour mener ses innombrables polémiques.

A partir du Moyen Âge, le rôle de la rhétorique sera de plus en plus réduit. Cela est dû au fait que la pensée scolastique va consacrer la logique aux dépens de la rhétorique. Les écoles des commentateurs et des glossateurs - sous l'influence de l'école d'Orléans ainsi que de Bartole et Balde - ont octroyé dès l'an 1300 à la dialectique platonicienne, fondée sur la diairesis et le syllogisme aristotélicien, le rôle de la meilleure méthode apte à interpréter le droit34. Cette approche avait été critiquée par le courant des juristes humanistes italiens représenté par Pétrarque qui sous l'influence de Cicéron 
et Augustin, défendaient le rôle de la rhétorique au sein de la science juridique 35 .

\section{La fracture entre la pensée critique et la rhétorique à l'aube de la modernité juridique}

\subsection{A. L'école du droit naturel moderne et l'abandon du débat}

A partir du XVI ${ }^{\mathrm{e}}$ siècle, le rôle occupé par la rhétorique au sein de la pensée juridique européenne va s'affaiblir sensiblement. Cela était dû au concours de différents facteurs tels que le cantonnement de l'art de la rhétorique à la fonction purement esthétique par de Pierre de la Ramée au XVIe siècle ainsi que par l'avènement de la philosophie de Descartes à partir du XVII' siècle. Selon Descartes la rhétorique n'était pas une méthodologie. Ce dernier affirmait ainsi dans son Discours de la méthode ce qui suit :

« J'estimais fort l'éloquence, et j'étais amoureux de la poésie ; mais je pensais que l'une et l'autre étaient des dons de l'esprit, plutôt que des fruits de l'étude $» 36$.

Selon Descartes la pensée critique se doit d'adopter uniquement des méthodes propres aux sciences dures, tel que le syllogisme scientifique.

Il est presque inutile de souligner que cette consécration du syllogisme a notamment eu une influence profonde sur la culture juridique européenne. Ceci dit, les juristes du XVIIe appartenant à la scolastique espagnole et à l'école moderne du droit naturel, avant même la parution de Discours de la méthode avaient déjà théorisé l’idée selon laquelle seul le syllogisme scientifique devait être appliqué au droit37. Ce fut ainsi que le théologien et juriste catholique Suarez soutenait l’idée que la loi naturelle ne pouvait être comprise par la raison humaine que par le biais de la méthode 
du syllogisme ${ }^{38}$. Quant à Grotius, ce dernier avait soutenu l'idée dans son ouvrage intitulé De jure belli ac pacis - publié en 1625, douze ans avant la parution du Discours de la méthode - que le syllogisme devait être l'unique type de raisonnement que devait adopter les juristes dans leur démarche.

Selon Grotius le droit se devait de se transformer en une science proprement dite. Pour cela le droit devait être appréhendé par le bais d'une démonstration de type géométrique (more geometrico) étant donné que le droit était perçu comme un système ordonné39. Donc Grotius affirmait que le droit devait être analysé selon la démarche déductive des mathématiques. La critique devait se faire, comme le remarque Goyard-Fabre « [...] en évitant toute dispute, éristique ou polémique [...]»40.

Le rationalisme de Grotius ainsi que la philosophie de Descartes ont notamment exercé une influence prépondérante sur l'œuvre des philosophes du XVIe siècle tels que Leibniz et Wolff. Leibniz voulait créer un langage pur et logique. Dans sa Dissertatio de arte combinatoria Leibniz théorisa une « écriture universelle » qui pourrait évincer toute dispute par le biais du calcul et ainsi :

«Il ne sera plus besoin entre deux philosophes de discussions plus longues qu'entre deux mathématiciens, puisqu'il suffira qu'ils saisissent leurs plumes, qu'ils s'asseyent à leur table de calcul [...] et qu'ils se disent l'un à l'autre : Calculons !»41

Les nouveaux philosophes du droit se devaient d'utiliser uniquement des raisonnements scientifiques et de délaisser les méthodes utilisées par les praticiens du droit, ce qui entrainait non seulement la mise à l'écart de la rhétorique mais aussi celle de la dialectique. 


\subsection{B. Vico et la critique de la pensée critique}

Le tournant rationaliste du droit inauguré par l'école moderne du droit naturel ainsi que la pensée critique de Descartes furent critiqués par le philosophe napolitain Giambattista Vico. Ce dernier avait développé une véritable contre-critique de la " pensée critique ». Une contre-critique qui aura pour pendant une redécouverte de la rhétorique ainsi que de la topique. Ce retour à la rhétorique s'explique par le fait qu'au début du XVIII ${ }^{\mathrm{e}}$ siècle à l'époque de Vico - la rhétorique était encore enseignée à l'Université de Naples, comme matière propédeutique à l'étude de différents domaines tels que la théologie, la médecine et, naturellement, le droit ${ }^{42}$. Avant de revenir sur la rhétorique de Vico, il convient de rappeler que l'enseignement de la rhétorique dans une université laïque, telle que l'Université de Naples, était une rareté pour le XVIII siècle. La rhétorique était enseignée avant tous dans les collèges religieux tels que les collèges jésuites. En effet, la Ratio studiorum qui était le programme d'études des collèges jésuites, en 1599, prévoyait l'enseignement obligatoire de la rhétorique et de la topique. Ce programme d'étude était enseigné dans les collèges jésuites de France et à partir du XVIII ${ }^{e}$ siècle dans les collèges du Québec43. Le manuel d'enseignement de la rhétorique dans le séminaire de la ville de Québec, rédigé par Urbain Boiret, en 1770, intitulé Rhétorica in Seminario Quebecensi data anno 1770 fait référence à l'art de la topique. Bernier explique que cet ouvrage repose sur une tradition rhétorique québécoise qui, selon lui, 
«[...] se caractérise par le souci d'établir l'argumentation sur une topique, c'est-à-dire sur une liste de lieux logiques d'où l'on puisse tirer à loisir des arguments généraux » 44 .

La rhétorique a joui d'une certaine importance au sein de la philosophie écossaise du XVIII ${ }^{\mathrm{e}}$ siècle. Cependant, tout comme en France et au Québec, la rhétorique, en Écosse, était une affaire de religieux. En effet, ce fut un révérend nommé Campbell, qui écrivit un traité sur la rhétorique intitulé The Philosophy of Rhétoric. Tout comme Vico, Campbell avait l'ambition d'élaborer une épistémologie fondée sur la rhétorique. Le révérend écossais soutenait l'utilité de l'enseignement de la rhétorique dans plusieurs domaines du savoir tels que les mathématiques, la médecine, l'économie et le droit45. Mais contrairement à Vico, Campbell se concentre uniquement sur l'aspect communicatif de la rhétorique et délaisse son aspect méthodologique, à savoir l'inventio et la topique ${ }^{46}$.

Vico, dans son traité critique intitulé De la méthode des études de notre temps, va remettre à jour la méthode de la topique. Dans cet ouvrage, Vico mène une critique de la pensée critique de Descartes. Une critique qui déploie des arguments semblables à ce que Cicéron avait utilisés à l'encontre de la dialectique stoïcienne dans ses Livres Topiques. Vico reproche à Descartes d'avoir bâti sa méthode critique en tenant compte uniquement de la phase du jugement critique et, par conséquent, d'avoir délaissé la phase de l'inventio, à savoir la phase topique. Le raisonnement décrit par Descartes repose, rappelons-le, sur une vérité première irréfutable (primum verum) qui échappe à toute discussion. Vico oppose à cette façon de procéder « critique » une méthode basée sur l'invention ou inventio, donc sur la recherche et la sélection d'arguments. Selon Vico, l'enseignement de la topique doit précéder celui de la critique. Tout comme les sophistes et les 
rhéteurs romains, Vico se préoccupait d'enseigner le sens critique à ses étudiants. Vico l'affirme clairement :

«[...] je serais donc d'avis d'enseigner aux jeunes gens tous les arts et les sciences en formant leur jugement de façon complète, afin que la topique enrichisse leur répertoire de lieux communs et que, tout en même temps, ils se fortifient, grâce au sens commun, dans la prudence et l'éloquence, et s'affermissent, grâce à l'imagination et à la mémoire, dans les arts qui reposent sur ces facultés de l'esprit. Qu'ils apprennent ensuite la critique, et qu'ils jugent alors [...] et avec leur propre jugement, les choses qu'on leur a apprises, et s'exercent à raisonner sur elles en soutenant les deux thèses opposées 47 ».

Vico avait saisi pleinement la dimension praxéologique du droit romain et de la façon dont le droit était enseigné :

« À Rome, par contre, les philosophes étaient les jurisconsultes euxmêmes, dans la mesure où ils plaçaient entièrement la sagesse dans la connaissance des lois telle qu'elle leur était acquise par l'expérience, [...],»48.

L'ouvrage de Vico intitulé De la méthode des études de notre temps représente une contre-critique de la pensée critique cartésienne ainsi qu'une tentative de réconciliation entre la rhétorique et la pensée critique. Une réconciliation qui était possible à travers une redécouverte de la rhétorique ainsi que de la topique cicéronienne.

\subsection{Une critique du droit sans rhétorique dans l'œuvre de Rousseau et Kant}

Vers la fin du XVIII ${ }^{\mathrm{e}}$ siècle, Rousseau et Kant fonderont une raison critique du droit qui se passera de l'art de la rhétorique.

Le Contrat social de Rousseau représente une véritable critique ou, mieux, un réquisitoire à l'encontre des dogmes de la pensée juridique. Le droit, selon Rousseau, se devait de s'affranchir de la pensée humaniste et du droit naturel classique (Aristote, Cicéron) tout comme des dogmes rationalistes de L'école du droit naturel moderne ${ }^{49}$. La critique permettait, 
selon Rousseau, de dévoiler les raisons historiques et politiques qui était sous-jacentes aux dogmes théorisés par les juristes ${ }^{50}$. Le rapport que Rousseau entretenait avec la rhétorique est complexe. Officiellement, l'auteur du Contrat social déclarait un certain mépris à l'égard de la rhétorique qui est définie dans l'Émile comme étant un « un pur verbiage ${ }^{51}$ ». Cela dit, la structure du Contrat social est influencée par la rhétorique. En effet Goyard-Fabre définit le Contrat social comme étant « [...] un exercice rhétorique parfait ${ }^{2}{ }^{»}$ et précise que la manière dont Rousseau dispose ses arguments « correspond à un art rhétorique raffiné qui est moins destiné à démontrer qu'à argumenter pro et contre $[. .] »$.53 .

La critique de Rousseau a notamment exercé une forte influence sur la philosophie critique du droit de Kant. Tout comme Rousseau, Kant est critique à l'égard de l'usage dogmatique de la raison telle que pratiquée par l'école du droit naturel moderne. Chez Kant la théorie de la critique devient une théorie du jugement, à savoir un «tribunal de la raison ». Ce tribunal tout de même se passera de l'art de la rhétorique que Kant assimile à celui de l'oratoire. Kant critiqua la rhétorique et l'oratoire dans sa Critique de la faculté de juger dans laquelle Kant classe l'oratoire parmi les « arts parlants » et donc « [...] l'art de mener à bien une opération de l'entendement comme s’il s'agissait d'un libre jeu de l'imagination (51) »54. L'oratoire et la rhétorique sont cantonnés à leur pure dimension esthétique. En dehors de cette dimension, la rhétorique devient, selon Kant, une « supercherie » $55 \mathrm{ou}$ même « [...] l'art d'abuser par la beauté de l'apparence (53) » à savoir « [...] une dialectique n'empruntant à la poésie que ce qui est nécessaire pour gagner les esprits avant même qu'il y ait exercice du jugement (53) [...]»56. Ainsi la rhétorique inhibe le sens critique. La rhétorique est donc à l'antithèse 
de la critique. La rhétorique n’a même pas sa place dans les tribunaux. Sur ce point Kant affirme clairement : (53) $\gg 57$.

« [...] on ne peut la conseiller ni pour les tribunaux, ni pour les chaires Kant affirme par la suite :

« Je dois avouer qu'un beau poème m'a toujours procuré une pure satisfaction, tandis que la lecture des meilleurs discours d'un orateur romain [...] s'est toujours mêlée pour moi du désagréable sentiment de désapprobation $[\ldots] » 58$.

Et conclut :

«Au reste un tel art, aussi bien à Athènes qu'à Rome, ne s'est élevé à ses sommets qu'à un moment où l'État courait à sa perte et où une vraie mentalité patriotique s'était éteinte $» 59$.

Cicéron incarne aux yeux de Kant la figure du vir dicendi peritus romain à savoir celle d'un « [...] orateur sans art, mais fertile en capacité d'impressionner [...] sans rester lui-même toujours fidèle à cet idéal »60.

Cette indifférence, voire même hostilité, que Kant avait à l'égard de la rhétorique sera critiquée par Grave et Jenisch. Ce dernier était un ancien élève de Kant qui était influencé par l'œuvre du révérend Campbell. Jenisch avait critiqué son ancien maître pour ne pas avoir pris en considération l’importance philosophique et pédagogique de la communication ${ }^{61}$.

Contrairement à Kant, Hegel avait eu une attitude plus nuancée à l'égard de la rhétorique. En effet, malgré le fait que la dialectique hégélienne demeurait une méthode qui n'accordait aucune place aux antilogies rhétoriques ${ }^{62}$ - étant celles-ci fondées uniquement sur la méthode classique des distinctions et des différentiations ${ }^{6}$ - Hegel avait reconnu cependant dans son Histoire de la philosophie une certaine importance au rôle que la pédagogie des sophistes fondée sur la méthode de l'antilogie avait eu dans l'évolution de la pensée philosophique ${ }^{64}$. 


\section{Le retour de la rhétorique}

A partir des années 1920, les historiens du droit tels que Vitton ${ }^{65}$, J. Stroux ${ }^{66}$ et Lanfranchi ${ }^{67}$ ont contribué - à travers l'étude des œuvres des rhéteurs romains - à la redécouverte de l'importance qu'avait revêtue la rhétorique au sein du droit romain.

Il faudra tout de même attendre la fin de la Seconde Guerre mondiale et la crise du positivisme juridique pour que la philosophie du droit se penche à nouveau sur la rhétorique et sur la topique. Ceci fut le cas d'abord de la nouvelle topique inaugurée par Théodor Viehweg durant les années 1950 et ensuite celui du courant de la nouvelle rhétorique de Chaïm Perelman.

Viehweg appartenait au courant juridique allemand de la Wertungsjurisprudenz. Un courant auquel appartenait, entre autres, des juristes allemands tels qu'Esser, Kriele, Larenz, Wieacker. Viewegh mena une critique au positivisme méthodologique qui dominait dans les facultés de droit allemandes jusqu'au début des années 1950 en s'inspirant de la pensée de Vico ${ }^{68}$. Dans sa thèse de doctorat intitulé Topik Jurisprudenz parue en 1953, Viehweg soutient l'idée selon laquelle la logique juridique demeurait une «pensée problématique » ou bien une «techné des Problemdenkens ${ }^{69}$ ", donc un art qui : « prend son point d'appui méthodologique dans le « problème ${ }^{70}$ ». Ainsi Viehweg s'opposait clairement à l'idée kelsenienne selon laquelle le droit pouvait résoudre tous les problèmes juridiques par le bais de la déduction logique. Viehweg, au contraire, soutenait que la logique juridique se distinguait de la logique axiomatique. Cet aspect singulier de la logique juridique s'expliquait par la tâche que le droit devait accomplir, à savoir résoudre des problèmes concrets. Tandis qu'à l'opposé la philosophie posait des problèmes sans pourtant 
proposer des solutions. Cette vision de la philosophie était influencée par la pensée d'Hartmann ${ }^{71}$.

La description que Viehweg donne du raisonnement juridique est clairement inspirée des Topiques de Cicéron et de l'ouvrage de Vico intitulé De la méthode des études de notre temps. C'est ainsi que selon Viehwegh reprend la division cicéronienne entre la phase topique (l'ars inveniendi) et la phase du jugement-critique ou ars iudicandi. Ceci étant, Viehweg, contrairement aux deux auteurs cités ci-dessus, ne cherchera pas à réconcilier ces deux phases. La problemdenken ou pensée problématique s'intéresse uniquement à la phase de la topique et délaisse la phase de la critique. Selon Viehwegh les antilogies présentes dans les différents arguments ou topiques peuvent à eux-seuls « [...] mener à la vérité » ${ }^{72}$. Ainsi la simple antilogie constitue en soi une critique autosuffisante et non pas une sélection préalable des arguments comme le souhaitait Cicéron.

Cette dichotomie entre la pensée problématique et la pensée critique avait à juste titre suscité les critiques de la part d'Apel et de Kriele. Ces deux auteurs ont soutenu que Viehweg en opposant la topique et la rhétorique à la pensée critique ait pris de Vico uniquement « [...] ce qui lui convenait »73, étant donné que le but de Vico était de réconcilier la topique avec la pensée critique par le bais de la rhétorique.

En ce sens, l'œuvre de Perelman a eu le mérite d'avoir réconcilié la rhétorique et la topique avec la dialectique. C'est ainsi que Perelman a développé une rhétorique critique. Dans son œuvre intitulée La logique juridique ce dernier définit ainsi cette nouvelle rhétorique : 
« La nouvelle rhétorique [...] ne se bornera pas, comme la rhétorique classique, à l'examen des techniques du discours public, [...] mais s'intéressera également au dialogue socratique, à la dialectique, telle qu'elle a été conçue par Platon et Aristote, à l'art de défendre une thèse et d'attaquer celle de l'adversaire, dans une controverse $» 74$.

Perelman a mené une critique du positivisme juridique en relançant l’intérêt envers la rhétorique. Tout comme Viehweg, Perelman récusait l’idée de l'existence d'une unique logique de type axiomatique déductive, qui soit applicable à toutes les sciences, y compris le droit. Dans son Traité de l'argumentation, Perelman rédige une topique d'arguments qui pouvaient être utilisés par des dialecticiens. Perelman puise ses recherches dans d'innombrables ouvrages de l'antiquité. Ces tópoi étaient des arguments ayant une portée persuasive et rationnelle. Ainsi Perelman affirme :

" Nous nous proposons d'appeler persuasive une argumentation qui ne prétend valoir que pour un auditoire particulier et d'appeler convaincante celle qui est censée obtenir l'adhésion de tout être de raison $» 75$.

Dans l'œuvre de Perelman, la redécouverte de la rhétorique a engendré la mise en valeur de la raison discursive et du caractère pratique de la logique juridique. Dès 1967 Perelman fonde le « Centre de philosophie du droit ${ }^{76}$ au sein duquel des juristes praticiens collaborent côte à côte avec des théoriciens. Dans son ouvrage intitulé Logique juridique. Nouvelle rhétorique, Perelman analyse le raisonnement juridique à partir d'une étude menée sur les arguments employés par les juges dans leurs jugements. Ainsi Perelman introduisait les méthodes des praticiens du droit dans la recherche universitaire.

C'est ainsi que Perelman réussit à réunir les éléments qui caractérisent la logique juridique. Cela dit, Perelman quant au rôle du juge ne confond jamais la tradition juridique continentale avec la Common Law. Perelman ne songe pas à un Juge Hercules tel que théorisé par Dworkin. Dans Logique juridique. Nouvelle rhétorique, Perelman affirme clairement 
que « [...] le rôle spécifique des juges est de dire le droit et non de le créer, quoique souvent l'obligation de juger, imposée au juge, l'amène à compléter la loi, à la réinterpréter et à l'assouplir »77.

Cette prise de conscience du caractère pratique du droit s'explique aussi par une connaissance approfondie que Perelman avait de l'histoire du droit et de la pensée juridique qui fit prendre conscience à Perelman de l'importance qu'avait revêtue la notion de controverse dans l'histoire du droit. Une opinion que Perleman partage avec Villey, Giuliani et Viehweg. Toujours dans Logique juridique. Nouvelle rhétorique, Perelman soutient que la controverse est une présence fixe dans l'histoire du droit et affirme ainsi ce qui suit :

« Nous constatons, en tout cas, aussi bien chez les juristes romains que chez les talmudistes ou les glossateurs de l'école de Bologne, que le droit s'élabore à travers les controverses et les oppositions dialectiques, les argumentations en sens divers. Il était bien rare que le raisonnement juridique puisse aboutir, comme dans les démonstrations mathématiques, à une conclusion contraignante ${ }^{78}[\ldots]$. La controverse avait pour effet, en premier lieu, d'exclure certains arguments, en montrant qu'ils n'étaient pas pertinents et en second lieu d'éliminer certaines solutions préconisées parce que déraisonnables, sans pourtant imposer nécessairement un genre d'arguments et une seule solution contraignante $\gg 79$.

Dans le système logique de Perelman les lieux communs (tópoi) jouent, dans l'argumentation, un rôle analogue à celui des axiomes dans un système formel ${ }^{80}$. Perelman définit le lieu commun comme étant :

« [...] un point de vue, une valeur, dont il faut tenir compte dans toute discussion et dont l'élaboration appropriée aboutira à une règle, une maxime, que l'orateur utilisera dans son effort de persuasion ${ }^{81}$.

Cependant, il existe entre une différence entre l'axiome et le lieu commun consistant en le fait que l'adhésion accordée aux lieux communs : « [...] n'est pas fondée sur leur évidence, mais au contraire sur leur ambiguïté, sur la possibilité de les interpréter et de les appliquer de façons diverses ${ }^{82}$. 
La philosophie du droit doit ainsi tenir compte de la dimension pratique du droit et par conséquent :

« La supériorité de la pensée juridique sur la pensée philosophique, c'est que, contrairement à celle-ci, qui peut se contenter de formules générales et abstraites, le droit est obligé d'envisager la solution des difficultés qui apparaissent quand il s'agit d'appliquer ces formules générales à la solution de problèmes particuliers $» 83$.

La rhétorique est aussi une pédagogie critique. Perelman a conçu un nouveau type d'enseignement du droit. Un enseignement fondé sur une simulation de controverse qui se déroule entre l'enseignant et les étudiants ${ }^{84}$. Comme l'explique Frison-Roche au sujet de la méthode d'enseignement perelmanienne :

« [...] l'argumentation doit devenir la méthode d'enseignement même parce qu'elle agit sur les étudiants et non pas contre les étudiants. Le professeur, qui admet la controverse et reconnait que les étudiants peuvent avoir raison de leur point de vue, ne s'affaiblit pas ${ }^{85}$.

En conclusion, Perelman a mené une critique de la logique juridique par le biais d'une redécouverte de la raison argumentative. Une telle démarche a eu le mérite de démontrer que la critique du droit peut se faire aussi par le biais de raisonnements et méthodes propres au monde des praticiens du droit, telles que la rhétorique et la topique. La rhétorique est un point de jonction entre la théorie et la pratique du droit, qui permet au droit de se critiquer par ses propres moyens. Cela n'exclue pas à priori l'approche interdisciplinaire, au contraire la méthode de Perelman a contribué à une redécouverte de la dimension philosophique historique et sociologique du droit. Comme nous l'avons vu, c'est la rhétorique à avoir ouvert le droit à l'interdisciplinarité et cela dès l'antiquité. Cela dit, le rapport avec les autres disciplines est un rapport entre pairs.

La nouvelle rhétorique s'inscrit pleinement dans le phénomène de la redécouverte de la raison discursive qui a vu le jour à partir des années 1960 
au sein de la pensée philosophique ${ }^{86}$. En ce sens la nouvelle rhétorique présente plusieurs points en commun avec la critique du droit d'Habermas, à savoir avec la théorie de l'agir communicationnel ainsi que l'éthique de la discussion. A première vue, cela n'est pas évident. En effet Habermas avait réduit la rhétorique à un rôle instrumental de l'utile à résoudre " des problèmes précis ${ }^{87}$. Mais, tout de même, une analyse approfondie de Habermas et Perelman nous induit à penser qu'entre les deux théories il peut s'établir un rapport de complémentarité. Les vérités auxquelles font référence les deux philosophes ne sont pas des vérités absolues, mais des vérités partagées basées sur un consensus. En ce sens, comme remarque Rigotti, la notion de dialogue universel d'Habermas est superposable à celle d'auditoire universel de Perelman ${ }^{88}$. Et comme l'a si bien dit Haarscher, les conditions de la discussion auxquelles Habermas a réfléchi dans son éthique de la communication correspondent « à la fondation philosophique que Perelman n'a pas eu le temps de donner [...] ${ }^{89}$. Chez ces deux auteurs, l'élément du débat joue un rôle central. Habermas affirme clairement dans son article intitulé Théorie relatives à vérité, que cette vérité fondée sur un accord peut être obtenue uniquement par le biais de la force de « l'argument meilleur ${ }^{90}$. Les philosophes de l'école de Francfort tels que Habermas et Apel ont la conviction que le droit tout comme la politique sont des pratiques (praxis) discursives et argumentatives ${ }^{91}$. Une raison discursive qui assume au sein de la pensée d'Habermas le statut de méthodologie critique du droit. Cet aspect a été mis en évidence par Melkevik. Ce dernier souligne que le modèle communicationnel d'Habermas pourrait « [...] constituer une théorie $\mathrm{du}$ jugement [...] ${ }^{92}$ cela par le biais d'un « $[. .$.$] tribunal$ communicationnel »93 qui valide des énoncés qui font l'objet de discussions théoriques et pratiques 94 ». Melkevik remarque encore que cette discussion 
sert aussi à « [...] évaluer et à critiquer contrefactuellement les pratiques quotidiennes »95. C'est un modèle qui laisse, toujours selon Melkevik, « [...] une place aussi bien à la tradition qu'à la raison dans son rôle de critique des traditions ${ }^{96}$. La théorie de l'agir communicationnel est une théorie critique qui considère le droit comme un " objet de lutte ou de controverse (polémique) avant de devenir sujet irénique »97. Ainsi, tout comme Perelman, Habermas a réconcilié la pensée problématique avec la pensée critique.

Selon Habermas, la critique et la raison discursive reposant sur une éthique de la discussion demeurent deux éléments qui peuvent exister uniquement au sein d'une démocratie participative ainsi que d'un État de droit. La raison discursive tout comme la rhétorique puise ses racines au sein de la démocratie athénienne ainsi que dans le droit romain. En ce sens, ces éléments demeurent les symptômes du niveau de santé démocratique d'un système politique. Bobbio avait saisi cet aspect dès la première édition du Traité de l'argumentation paru en Italie en 1966. Bobbio, qui en avait rédigé la préface, souligne que les techniques argumentatives décrites dans l'ouvrage de Perelman et Olbrechts-Tyteca peuvent avoir lieu uniquement dans un système politique et juridique qui admet la discussion libre. Bobbio accorde un caractère rationnel à la raison argumentative. Celle-ci à son dire ne peut exister qu'au sein d'une société d'individus libres de mener des choix rationnels ${ }^{98}$. Ce sont des individus qui ont conservé la faculté de jugement et donc leur faculté critique. Bobbio conclut que la raison argumentative est le meilleur antidote contre les fanatismes de tous genres99. En ce sens, la position de Bobbio se rapproche de l'opinion que Gramsci avait exprimée à l'égard de la rhétorique. Gramsci, avant même Perelman, avait préconisé un rapport de complémentarité entre la dialectique et la rhétorique ${ }^{100}$. En ce 
sens Gramsci se rapproche de la thèse que Perelman a développé à partir les années 1960 .

\section{Conclusion}

Pour conclure, la rhétorique représente un instrument utile à la critique du droit. Comme nous avons pu le voir, cet art est à l'origine de la logique juridique ainsi que de la critique du droit. Perelman nous enseigne que la dichotomie entre la rhétorique et la pensée critique n'as pas et n'a plus raison d'être. La rhétorique est par définition une pensée critique. Une critique qui est avant tout une autocritique et cela à travers le raisonnent du pro et contra qui s'avère être un moyen de sélection d'arguments. La rhétorique aide le droit à développer une critique du droit interne au monde du droit. Une critique du droit qui ne remet pas en cause les méthodes de raisonnement utilisées par les praticiens du droit et qui ne renonce pas à voir dans le modèle du procès accusatoire une source d'inspiration.

En effet, comme nous l'avons vu plus haut, la rhétorique avec son éthique du discours et sa raison argumentative ne peut se développer pleinement qu'au sein d'un État de droit et dans une démocratie participative. Les juristes doivent tenir compte de l'ancienneté et de la richesse de leur histoire. C'est en se connaissant soi-même que le droit peut affronter le défi de la modernité.

* Amar Laidani, doctorant en «Histoire du droit» auprès de l'Université de Montpellier en cotutelle avec l'Université Laval; e-mail : amar.laidani.1@ulaval.ca 1 Maurizio MANZIN, "In principio era il contraddittorio", La giustizia penale, 51, 2010, pp. 397-406.

2 Michel Villey, Philosophie du droit. Définitions et fins du droit. Les moyens du droit, Paris, Dalloz, p. 209. 
3 Eugenio RIPEPE, «Civiltà giuridica europea e retorica giudiziaria: un'anteprima a teatro», dans (dir.) Maurizio

MANZIN, Federico PUPPO, Audiatur et altera pars. Il contraddittorio fra principio e regola, Milano: Giuffré, 2008,

pp. 63-95.

4 Carlo ROVELli, ANAXIMANDRE de Milet ou la naissance de la pensée scientifique, traduit de l'italien par Matteo SMERLAK, Paris, Dunod, 2009, 2015 pour la nouvelle présentation, p. 74 .

5 Werner JAEGER, Paideia, la formation de l'homme grec Vol. I, La Grèce archaïque, Le génie d'Athènes, traduit de l'allemand par André et Simone DEVYER, Paris, Gallimard 1964, p. 198.

6 Pierre GuERIN, L'idée de justice dans la conception de l'univers chez les premiers philosophes grecs, Paris, Librairie Félix Alcan, 1934.

7 Jean BRUN, Héraclite : La philosophie de l'Éternel Retour, Paris, Éditions Seghers, 1965, p. 126.

8 Laurent PERnot, La Rhétorique dans l'Antiquité, Paris, Libraire Générale Française, 2000, p. 25.

9 Jacqueline DE Romilly, Les Grands Sophistes dans l'Athènes de Périclès, Paris, Éditions de Fallois, 1988, p. 65.

${ }^{10} \mathrm{Id} ., \mathrm{p} .146$.

${ }^{11} \mathrm{Id}$.

${ }^{12}$ Luc Bresson, « Les Sophistes », dans Monique CANTO-SPERber (dir.), Philosophie grecque, Presses Universitaires de France, Paris 1997, pp. 112-116.

${ }_{13}$ Franco Todescan, Metodo diritto politica. Lezioni di storia del pensiero giuridico, Bologna, Monduzzi Editore, 2012, p. 22.

${ }^{14}$ L. PERNOT, préc., note 8, p. 76.

${ }^{15}$ Aristote, Rhétorique, présentation de Michel MeYer et commentaire de Benoit Timmermans, traduit du grec par Patricia VANHEMELRYCK, Paris, Librairie Général Française, collection Les classiques de la Philosophie, 1991.

16 Antonino FALlone, Il processo aperto: Il principio di falsificazione oltre ogni ragionevole dubbio nel processo pe-nale, préface de Giuseppe BENTIVEGNA et Delfino Siracusano, Milano, Giuffré, Collection Osservatorio Perma-nente Sulla Criminalità, Organizzata, 2012.

17 Aldo Schiavone, Ius. L'invention du droit en Occident, traduit de l'italien par Geneviève et Jean Bouffartigue, Paris, Éditions Belin, collection l'antiquité au présent, 2008. Titre original, Ius. L'invenzione del diritto in Occidente, Torino, 2005.

${ }_{18}$ Id., p. 171.

19 Id., p. 209.

${ }^{20}$ Id., p. 196.

${ }^{21}$ Alain MicheL, Les rapports de la rhétorique et de la philosophie dans l'œuvre de CICERON. Recherches sur les fondements philosophiques de l'art de persuader, 
Éditions Peeters, Paris-Louvain, Bibliothèque d'Études Classiques, $2^{\mathrm{eme}}$ édition, 2003, p. 463, (réimpression de la thèse de doctorat paru en 1960 pour les Édition de la Sorbonne).

${ }_{22}$ Guido FASsò, Storia della filosofia del diritto-Vol.-I Antichità e medioevo, Roma, Editori Laterza, 2001, p. 103.

${ }_{23}$ A. Michel, préc., note 21, p. 461.

24 Ciceron, « Des lois » dans CiCERon, De la République ; Des lois, traduit du latin par Charles APPUHN, Paris, Flammarion, 1965.

${ }_{25}$ A. Michel, préc., note 21, p. 463.

${ }^{26}$ CiCERON, « L'orateur », traduit du latin par Michel Alphonse AgNANT, dans CICERON œuvres complètes, tome 32, Paris, Panckoucke Éditeurs, 1839.

27 CiCERON, "Les Topique », traduit du latin par MiChEL Damas HinARD, dans Euvres complètes de CICERON (dir.) MICHEL NISARD, Tome I, Paris, Chez Firmin Didot frères, fils, Libraires, 1869.

${ }^{28}$ M. VILLEY, préc., note 1, p. 200.

29 Id.,p. 210.

3o Giovanni NEgRI, «Cicerone come fonte di cognizione del diritto privato. L'esempio della causa curiana appunti per una ricerca Cicerone come fonte di cognizione del diritto privato. ", dans Atti del XIII Colloquio Tullianum, Ciceroniana, Rivista del Centro Studi Ciceroniani, Nuova Serie Band, V. XIII. Milano, 2009, p. 182.

${ }_{31}$ Tertullien, Apologétique, traduit du latin par Jean-Pierre WALtzing et Albert Severyns, Deuxième édition, Paris, Société d'Edition « Les Belle Lettres », 1961.

$3^{2}$ LACTANCE, Institutions Divines Livres V, Tome l, traduit du latin par Jean MonAT, Paris, Les Éditions du Cerf, 1973.

33 Henri-Irénée Marrou, Saint-Augustin et la fin de la Culture Antique, Paris, Éditions De Boccard, 1958, p. 519.

34 Maurizio MANZIN, « La questione retorica alle origini dell'umanesimo giuridico », dans Francesco CAVAlLA (dir.), Retorica processo e verità, Padova, CEDAM, 2005, p. 129.

35 Maurizio MANZIN, Il petrarchismo giuridico. Filosofia del diritto. Filosofia e logica del diritto agli inizi dell'umane-simo, Padova, CEDAM, 1994, p. 994.

${ }^{36}$ Renée DesCarTes, Discours de la méthode, Paris, Édition Gilson VRIN, 1976, p. 7.

37 Simone GOYARD-FABre, Critique de la raison juridique, Paris, Collection Thémis Philosophie, Presses Universitaire de France, 2003, p. 19.

$3^{8}$ F. TODESCAN, préc., note 13, p. 102.

39 Simone GoYARD-Fabre, Critique de la raison juridique, Collection Thémis Philosophie, Paris, Presses Universitaires de France, 2003, p. 18.

40 Id., p. 19.

${ }^{41}$ Louis CoutURAT, La logique de LEIBNIZ, d'après des documents inédit, Hildesheim, George Olms Verlag AG, p. 98, ouvrage cité dans la note 3. 
42 Giambattista Vico, La méthode des études de notre temps. (De notri temporis studiorum 1708), préfacé et traduit de l'italien par Alain PoNs, en ligne http://www.mcxapc.org/docs/conseilscient/0511vico_pons.pdf (site consulté le 16 août 2017), p. 07.

43 Lettre du P. Jospeh GERMAIN touchant la mission canadienne en l'année 1711, citée dans l'ouvrage de Yvan LAMONDE, La philosophie et son enseignement au Québec (1665-1920), Québec, Cahier du Québec, 1980, p. 26, note 1.

44 Marc-André BernIER, Portrait de l'éloquence au Québec (1760-1840) Sainte Foy, Québec, La Presse de L'Université Laval, 2002, p. 415.

45 Christine DE Gemaux, "Crise épistémologique du discours de KANT au romantisme allemand. La rhétorique, méditation entre logique et esthétique ? ", dans Charlotte MOREL (dir.), Esthétique et logique, Villeneuve d'Ascq, Presses Universitaires, 2012, p. 234.

${ }^{46}$ Isabelle BouR, «Au-delà de la rhétorique. The Philosophy of Rhetoric de George CAMPBEll ", dans Pièrre MORERE (dir.), Écosse des Lumières. Le XVIII siècle autrement, Grenoble, Ellug, 1997, p. 58.

47 G. VICO, préc., note 42, p. 48.

48 Id., pp. 70-71.

${ }^{49}$ S. GOYARD-FABRE, préc., note 36, p. 57.

5o Id., p. 62.

${ }^{51}$ Jean Jacques Rousseau, Euvres complètes, Tome deuxième, Paris, Chez Furne, Libraire-Éditeur, p. 556.

${ }^{2}$ Simone GOYARD-FABRe, Politique et philosophie dans l'œuvre de Jean-Jacques ROUSSEAU, Paris, PUF, 2001, p. o3.

$53 \mathrm{Id}$.

54 Emmanuel KANT, Critique de la faculté de juger, traduit de l'allemand par Alain Renault, Paris, Flammarion, 1995, p. 308.

55 Id. p. 315, note 1.

${ }^{56} \mathrm{Id}$.

${ }^{57}$ Id., p. 314.

${ }^{8}$ Id., p. 315.

$59 \mathrm{Id}$.

6o Id.

${ }^{61}$ Christine DE GEMAUX, « L'élève et le maitre. D. JENISCH et E. KANT face à la crise de la tradition rhétorique », dans M-Grimberg, M-T Mourey, E. Rothmund. W. SABleR, A.-M. SAINT-Gille et M. SilhouetTe (dir.), Recherches sur le monde germanique: regards, approches, objets, Paris, Presse de l'Université de la Sorbonne, 2003.

62 Errol. E. HARRIS, Lire la logique de HEGEL. Raison dialectique, Lausanne, L'Age d'homme, 1987, p. 59

$63 \mathrm{Id}$. 
64 Georg Wilhelm Friedrich HEgEL, Leçons sur l'histoire de la philosophie, traduction de l'allemand par Pierre GARNIRON, Paris, Vrin, 1985, p. 244

65 Paolo VitTon, I concetti giuridici nelle opere di Tertulliano, Roma, Tipografia della R. Accad. Nazionale dei Lincei, 1924.

66 Johannes Stroux, «Summum ius iniuria. Ein Kapitel aus der Geschichte der Interpretatio iuris», in Festschrift Speiser Sarasin, Leipzig, 1926.

${ }_{67}$ Fabio LANFRANCHI, Il diritto nei retori romani, Milano, Giuffré, 1938, p. 08.

68 Lutz GELDSETZER, "Il metodo degli studi di Vico e la giurisprudenza tedesca », dans Bollettino del Centro di Studi vichiani XXII-XXIII (1992-1993), p. 369.

69 Peter Degat, Littératures contemporaines sur la "topique juridique", préface de Michel Villey, Paris, Presses Universitaires de France, Paris, 1981, p. 1.

70 Id.

${ }^{71}$ Id., p. 370.

72 Theodor Wienweg, Topik und jurisprudenz, München, C H. Becksche Verlagsbucggandlung, 1953, p. 24.

73 P. DEGat, préc., note 69, p. 29.

74 Chaïm Perelman, Logique juridique. Nouvelle rhétorique, Paris, Dalloz, Deuxième édition, 1979, p. 108.

75 Chaïm Perelman, Lucie Olbrechts-Tyteca, La nouvelle rhétorique. Traité de l'argumentation, Paris, Presses Universitaires de France, 1958, p. 36.

${ }^{76}$ Benoit Frydman, « PERELMAn et les juristes de l'École de Bruxelles », dans Benoit Frydman, Michel MeYer (dir.), Chaïm PERELMAN. De la nouvelle rhétorique à la logique juridique (1912-2012), Paris, Collection L'interrogation philosophique, Presses Universitaire de France, 2012, p. 233.

77 C. PERELMAN, préc., note 71, p. 154.

${ }_{78}$ Id. p. 07.

79 Id.

$80 I d$.

${ }^{81} I d .$, p. 118.

$82 I d$.

83 Id., p. 121.

84 Marie-Anne FrISON-RochE, « Le modèle perelmanien au regard des méthodes d'enseignement du droit », dans Benoit Frydman, Michel Meyer (dir.), Chaïm PERELMAN. De la nouvelle rhétorique à la logique juridique (1912-2012), Paris, Collection L’interrogation philosophique, Presses Universitaires de France, 2012, p. 227.

85 Id.

${ }^{86}$ Richard M. RoRTY, The linguistic Turn, Chicago, University of Chicago Press, 1967, p. 28.

87 Jürgen HABERMAS, Le discours philosophique de la modernité. Paris, Gallimard, 1988, p. 177. 
88 Francesca RigotTi, La verità retorica: etica, conoscenza e perusasione, Milano, Feltrinelli, 1995, p. 2.

89 Guy HAARSCHER, " Apres PERELMAN, dans Justice et argumentation », dans Guy HAARSCHER, Léon INGBer (dir.), Essais à la mémoire de Chaïm PERELMAN, Bruxelles, Édition de l'Université de Bruxelles, 1986, p. 225.

90 Jürgen HABERMAS, "Théories relatives à la vérité ", in Logique des sciences sociales et autre essais, traduit de l'allemand par Rainer Rochlitz, Paris, PUF, coll. «Philosophie d'aujourd'hui », 1987, p. 308.

91 Herman PARRET, " Au-delà de la rhétorique du juridique: justifier par l'éthique, légitimer par l'esthétique », dans Droit et société, Le discours juridique. Langage, signification et valeurs, n. 8, 1988, p. 76.

92 Bjarne MelKevik, Droit et Agir Communicationnel : Penser avec HaberMaS, Paris, Buenos Books International, 2012, p. 26.

93 Id.

94 Id.

95 Id., p. 27.

${ }_{96} \mathrm{Id}$.

97 Id., p. 93.

98 Chaİm Perleman, Lucie Olbrecht Tyteca, Trattato dell'argomentazione, préfacé par Norberto Bоввіо, Milano, Einaudi, 1966, p. 17.

99 Id., p. 18.

100 Antonio Gramsci, Il materialismo storico (Q.11), Roma, Editori Riuniti, 1979, p. 71 . 